\title{
Expansion of Models for Heart Rate Variability beyond the Autonomic Nervous System
}

\author{
Jennifer Straub ${ }^{1 *}$, Martin Bachler ${ }^{2}$ \\ ${ }^{1}$ Institute of Analysis and Scientific Computing, TU Wien, \\ Wiedner Hauptstraße 8-10,1040 Vienna, Austria; *jennifer.straub@tuwien.ac.at \\ 2 AIT Austrian Institute of Technology $\mathrm{GmbH}$, Center for Health \& Bioresources, Biomedical Systems, \\ Giefinggasse 4, 1210 Vienna, Austria
}

SNE 29(4), 2019, 189-198, DOI: 10.11128/sne.29.tn.10494

Received:Sept. 15, 2019; Revised: November 5, 2019;

Accepted: November 10, 2019

SNE - Simulation Notes Europe, ARGESIM Publisher Vienna, ISSN Print 2305-9974, Online 2306-0271, www.sne-journal.org

Abstract. According to the World Health Organisation, diseases of the cardiovascular system (CVS) are currently the main cause of death all over the world. Therefore, their understanding, prediction, and prevention with the help of non-invasive, cost effective methods is of great interest. Analysis of the heart rate and its change over time can give valuable insight into the health status of a patient, and is easily derived from electrocardiogram (ECG) data. Reduced heart rate variability (HRV) is associated to an increased probability of dying after myocardial infarctions and indicates inflammatory processes. It is symptomatic of mental disorders such as depression and burn-out. Different approaches in modeling and simulation of HRV can provide new insight into the nonlinear interplay of cardiovascular regulation. In this work, three models for HRV are implemented and compared. They include the firing rate of the baroreceptors, respiration, activity of the sympathetic and parasympathetic nervous system, stroke volume, cardiac noradrenaline and acetylcholine concentration, as well as a windkessel model including peripheral resistance and arterial compliance. First, an existing model for HRV based on respiration and baroreflex activity was implemented and analyzed. A second model was created through adaption of the first model. Based on a model for the autonomic response to orthostatic stress, a third model was implemented as well. All models were realized in Simulink 2017b, and their validation is performed based on 60 five-minute ECG recordings from 30 subjects. The simulation results are compared to subject data based on the standards of HRV measurement by the Task Force of the European Society of Cardiology and the North
American Society of Pacing and Electrophysiology. Each of the three modeling approaches showed specific advantages, disadvantages, and possibilities for further improvement. The results provide basis for extension of HRV models, paving the way for the future usage of model prediction in the field of cardiovascular diseases.

\section{Introduction}

Heart rate variability (HRV), the change in time intervals between successive heart beats, reflects the activity of the autonomous nervous system, and gives information about the overall condition of the cardiovascular system. The heart rate (HR) keeps on changing as a result of non-static physiological and psychological regulatory mechanisms, which interact in a non-linear way. HRV is a sign of a healthy cardiovascular system. On the contrary, a reduced change between adjacent interbeat times can indicate health problems such as coronary artherosclerosis, inflammation, and depression. HRV decreases with age, and depends on the HR, since a higher HR results in shorter interbeat intervals, and therefore leaves less time for changes [1].

As HRV can be easily derived from electrocardiogram (ECG) data and therefore is a simple and noninvasive diagnostic tool, great interest lies in attaining a deeper understanding of its nonlinear regulatory mechanisms, using modeling and simulation. Creating a model which is complex enough to reproduce important characteristics of HRV, but still simple enough to maintain applicability, can lead to a deeper physiological insight [2], opening up new possibilities for diagnostics and therapy [3]. 


\section{Background}

\subsection{Controlling Mechanisms of the Cardiovascular System}

The Autonomic Nervous System The autonomic nervous system (ANS) has two antagonistic branches: the sympathetic and the parasympathetic nervous system. The sympathetic system is mainly activated during exercise and emergency situations to increase HR and blood flow. The parasympathetic system is dominant during rest conditions. It regulates basic body funcions and decreases the HR [4]. The excitement of their effector organs happens via the release of acetylcholine or noradrenaline [5].

Efferent nervous activity of the ANS is mostly regulated by autonomic reflexes, for example the baroreceptor reflex[4]. The tissues of the heart are innervated by both systems, but parasympathetic fibres are mainly distributed to the atria, the sinus node, the AV-node, and not to the ventricles. Through sympathetic stimulation, noradrenaline is released regardless of the stretch of the muscle fibres, causing stronger contractions, as well as faster depolarisation and relaxation, resulting in a higher HR. Parasympathetic activity antagonistically prevents the release of noradrenaline from sympathetic nerves. The parasympathetic HR reduction also comes from a release of acetylcholine at the heart's pacemaker cells. The release of sympathetic noradrenaline also causes the blood vessels in the body to contract, which regulates blood pressure [6].

Continuous discharge of sympathetic nerve fibres increases pumping by 30 percent compared to no sympathetic stimulation. The average HR for young adults of 70 beats per minute (bpm) can be increased up to approximately $200 \mathrm{bpm}$ by strong sympathetic stimulation, while simultaneously intensifying the contraction to as much as double normal, increasing the amount of blood pumped and augmenting the ejection pressure [6].

The Baroreflex The baroreceptors are nerve endings located at the sinus caroticus and in the aortic arch. They almost immediately generate nervous signals when stretched by high blood pressure. The signals are then forwarded to the brain stem. From there, secondary signals increase the activity of the parasympathetic center. This leads to a decrease in HR, a weaker heart contraction, and vasodilation.
Conversely, low blood pressure has the opposite effect, causing oscillation of the baroreceptor reflex. If arterial blood pressure is chronically increased or decreased, baroceptors activity changes at first, but adapts to the new pressure level in 1 to 2 days[4].

The Arterial Windkessel Effect The wall of the thoracic aorta contains a lot of elastic fibres. Therefore, the blood ejected from the heart during systole causes the aorta to stretch, which reduces the systolic pressure rise and subsequently has an effect on the baroreceptors. The aorta then returns to its original shape, pushes the blood further into the arterial system and ensures a rather continuous blood flow [5].

The Effect of Respiration on the Heart Respiratory sinus arrhythmia (RSA) is the synchronous variation of HR and respiration. The stroke volume of the heart is almost equivalent to the blood volume in the capillary bed, and as a consequence, variation of the HR during inspiration and expiration affects the efficiency of the gas exchange[7].

During inspiration, the intervals between successive heartbeats are shortened, whereas during expiration longer intervals are observed. RSA can be influenced by cardiopulmonary function, sleep or wakefulness, age, and many other factors [7].

\subsection{State of the Art}

Existing models include various components of the cardiovascular system and make use of highly statistical approaches [8,9], as well as differential equations [10], and discrete events [11], but a lack of detailed physiological reasoning is found in a lot of them.

There is broad consensus about the physiological reasonableness of the integral pulse frequency modulation (IPFM) model with constant or varying threshold for the generation of a heart beat time series [12, 13].

A simplistic approach for including the effect of respiration on the HR is presented by Brennan et al. [14]. Although this approach is physiologically reasonable, respiratory influences on the HR are usually included into the parasympathetic activity[15].

Aside from respiration, the baroreflex is often part of HRV models. Olufsen et al. present a model of baroreflex regulation of the HR during orthostatic stress, based on different types of baroreceptors [16]. 
Instead of including more effectors than the baroreflex, Ursino proposed a compartment model, which includes a more detailed description of the left and right heart, the pulmonary circulation, and their respective resistances and compliances, which are modulated by the activity of the ANS [17].

DeBoer, Karemaker, and Strackee [11] created a more extensive model based on difference equations, amongst other factors including an RR-interval dependent contractility of the myocard.

A closed loop model, which includes respiration, the baroreceptor reflex, ANS activity, a windkessel time constant, contractility, and even neurotransmitters levels, is proposed by Seidel and Herzel. Opposite to the previously mentioned model, it combines discrete signals and continuous ones. A unique characteristic of this model is the use of a phase response curve, which modulates the input of the parasympathetic nervous system depending on its time of occurrence during the heart cycle [2].

\section{Methods}

Integral Pulse Frequency Modulation Model Many authors assume a modulating effect of the ANS activity on the sino-atrial node[12]. This influence is summarized as a function $m(t)$ with zero mean and a rather small amplitude. The beat occurrence times $t_{k}$ generated by the Integral Pulse Frequency Modulation (IPFM) Model can then be written as

$$
k=\int_{0}^{t_{k}} \frac{1+m(t)}{T} d t \quad k=1,2,3 \ldots
$$

Poincaré Plots Poincaré plots allow the geometrical analysis, and quantification of HRV by plotting each RR-interval against the following one, resulting in a scatterplot. It simultaneously gives an overview of the overall, short and long term beat-to-beat variability[18]. To characterize the shape of a Poincaré plot, the ellipse fitting technique is used. Other Statistical measures for short and long term variability are SD1 and SD2 as described by Brennan [19].

\section{Models}

\subsection{Model 1: An HRV model including respiration and baroreflex}

As a first nonlinear model for HRV, presented by Henrik Seidel and Hanspeter Herzel including baroreceptors, ANS activity, neurotransmitters, contractility, vasoconstriction, and a windkessel, depicted in Figure 1, was implemented. All parameters were chosen according to the original publication [2].

\subsection{Model 2: An Adapted Seidel and Herzel Model}

The following paragraphs describe a second model, based on the previously presented one, but with the aim of creating a more physiologically accurate model.

Blood pressure and systolic duration The blood pressure curve generated by the previous model shows a sharp peak at the end of the systole, followed by a steep exponential decay. If ever, a discontinuity in the time derivative of the blood pressure function, as seen at the end of the systole, should occur when the aortic valves close due to a lack of output from the left ventricle. The systolic duration $\tau_{s y s}$ was estimated at $125 \mathrm{~ms}$, which is clearly too short compared to results from in vivo studies, and was therefore set to $300 \mathrm{~ms}$ [20]. The original equation for systolic pressure was replaced by the following:

$$
p_{I}=d_{i-1}+S_{i}\left(\frac{1.8\left(t-t_{i}\right)}{\tau_{s y s}}\right) \exp \left(1-\frac{1.8\left(t-t_{i}\right)}{\tau_{s y s}}\right)
$$

Sympathetic activity The original equation for sympathetic activity was modified to avoid discontinuities in the first derivative. This leads to the following expression:

$v_{s}=\max \left(0, v_{s, 0}-k_{s, b} v_{b}+k_{s, r} \frac{\sin \left(\pi s_{f} t+\Delta \phi_{s, r}\right)+1}{2}\right)$.

A similar problem occurs due to the subtraction of $k_{s, b} v_{b}$. Therefore, a butterworth low-pass filter is introduced, using order 3 and a passband edge frequency of $0.15 \cdot 2 \pi \frac{\mathrm{rad}}{\mathrm{sec}}$. The filter output is multiplied by 0.4 , to keep a reasonable level of sympathetic activity. 


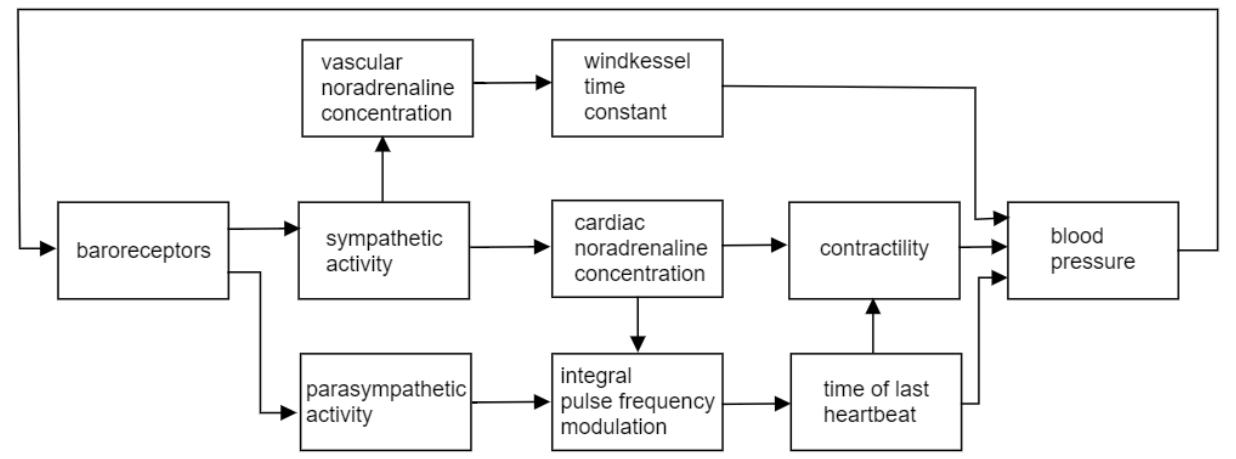

Figure 1: Schematic representation of the CVS components in Model 1 and 2.

Parasympathetic activity and respiration Respiration is only included in the parasympathetic activity [21]. Assuming that the breathing pattern is represented by a function $r(t)$, ranging from -1 at total expiration to 1 at total inspiration, a linear dependence of parasympathetic activity on respiration is assumed. To keep the same overall level of activation, the original equation was changed to

$$
\begin{aligned}
v_{s}=\max \left(0, v_{p, 0}\right. & +k_{p, b} v_{b} \\
& \left.+k_{p, r}\left(\frac{\sin \left(\pi p_{f} t+\Delta \phi_{p, r}\right)}{2}+\frac{r(t)}{2}\right)\right),
\end{aligned}
$$

including a basic parasympathetic frequency $p_{f}$.

Correction of the mean HR To accurately reproduce the mean HR defined by $T_{0}$, the product of the sympathetic and parasympathetic activities $f_{s}$ and $f_{p}$ should equal 1 in the long term as part of the IPFM input. To ensure this, the mean of $f_{s}(t) f_{p}(t)$ at time $t$ was divided by its mean over the previous $10 \mathrm{sec}$.

\subsection{Model 3: An HRV model with different baroreceptor types}

In this section, the model presented by Olufsen et al. is described and extended. It was designed to derive ANS activity and thus HR from measured blood pressure values during periods of orthostatic stress. Most parameters, such as $\tau_{S}, \tau_{I}, \tau_{L}, k_{S}, k_{I}, k_{L}$, and $M$ were derived from animal experiments, others were estimated empirically [16].
Baroceptors Olufsen et al. distinguish three types of baroreceptors. The model is based on the rate of change of mean arterial pressure $\bar{p}$ over time. Assuming that the pressure function $p(s)$ is continuous and bounded, by using the integral rule of Leibniz there holds:

$$
\frac{d \bar{p}}{d t}=\alpha\left(\frac{d}{d t} \int_{-\infty}^{t} p(s) e^{-\alpha(t-s)} d s\right)=\alpha(p-\bar{p}) .
$$

Three types of baroreceptors are defined as

$$
\frac{d n_{i}}{d t}=k_{i} \frac{d \bar{p}}{d t} \frac{n(M-n)}{(M / 2)^{2}}-\frac{n_{i}}{\tau_{i}} \quad i=S, I, L
$$

$\mathrm{S}$ stands for short, I for intermediate, and $\mathrm{L}$ for long time scales. They represent the variations in threshold for different baroreceptor types and are included in the equations via $\tau_{S}, \tau_{I}$, and $\tau_{L}$. The maximum firing rate is taken into account by $M$, which is set to 120 . For the overall firing rate $n$, there holds $n=n_{S}+n_{I}+n_{L}+N$ and $n_{S}, n_{I}$ and $n_{L}$ give the deviation from the mean firing rate $N$.

The autonomous nervous system The parasympathetic activity $T_{p}(n)$ is defined as $n$ divided by $M$. The sympathetic activity includes a time-delay $\tau_{d}$ and is given as:

$$
T_{s}(n)=\frac{1-n\left(t-\tau_{d}\right) / M}{1+\beta T_{p}(n)} .
$$


The Heart as effector of the ANS Cardiac noradrenaline and acetylcholine concentrations are calculated as

$$
\frac{d C_{n o r}}{d t}=\frac{-C_{n o r}+T_{s}}{\tau_{n o r}}, \frac{d C_{a c h}}{d t}=\frac{-C_{a c h}+T_{p}}{\tau_{a c h}},
$$

and to generate an input for the IPFM, they were combined with the mean $\mathrm{HR} H_{0}$ giving

$$
\frac{d \varphi}{d t}=H_{0}\left(1+M_{s} C_{n o r}-M_{p} C_{a c h}\right) .
$$

In order to create a closed loop, the previously presented model had to be further expanded.

The Arterial Windkessel A three-element windkessel model was chosen to generate a pressure curve as input for the baroreceptors. It is dependent on total arterial compliance $C$, peripheral resistance $R$ and input impedance $R_{c}$ and was chosen accoring to Westerhof et al. [22].

In order to solve the given differential equation, a function for the flow from the aortic root $q(t)$ was chosen based on findings in [23] as

$$
q(t)=\exp \left(2\left(1-\frac{1}{t_{s}}\right)+\frac{1}{\left(\frac{2 t}{t_{s}-1}\right)^{2}-1}\right) \frac{S V}{I}
$$

The systole duration in ms is calculated as $t_{s}=540-$ 2.1. $H R$ according to [24]. $S V$ is the stroke volume in milliliters eqaling $n=0.5+0.5 \frac{R R_{n-1} V_{\text {ref }}}{1000}$ according to [25]. At $60 \mathrm{bpm}$ it was assumed to be $70 \mathrm{ml}$. $I$ equals the integral of the exponential term in $q(t)$ over the interval $\left[0 ; t_{s}\right][23]$.

Autonomic nervous system activity Two sinusoidal functions with frequencies $f_{p}$ and $f_{s}$ were added to the model, giving

$$
\begin{gathered}
T_{p}(n)=\frac{n(t)}{M}+C_{p} \sin \left(2 \pi f_{p}\right), \\
T_{S}(n)=\frac{1-\frac{n\left(t-\tau_{d}\right)}{M}+u(t)}{1+\beta \frac{n(t)}{M}}+C_{S} \sin \left(2 \pi f_{s}\right) .
\end{gathered}
$$

To avoid fast fluctuations in sympathetic nervous system activity, a butterworth filter of order 3 and passband edge frequency of $0.15 \cdot 2 \pi$ was applied to $T_{S}(n)$.
IPFM and Respiration The ANS input of the IPFM model was scaled analogously to the second model. The respiration was included by using a varying threshold for the IPFM, as proposed by Barbi et al. [26]. The threshold is chosen as $i(t)=1+\frac{r(t)}{20}$ with $r(t)$ oscillating between -1 and 1 , depending on the breathing cycle.

\section{Simulation}

All three simulations were implemented in Simulink $2017 \mathrm{~b}$ and the RR-intervals were further analysed in Matlab 2017b.

RR-interval data from 30 subjects with essential hypertension was used to derive parameters for the simulation. One of six different guided breathing patterns was used for each subject. During the 10min breathing exercise, an ECG was recorded at a sampling rate of 256 $\mathrm{Hz}$ [27].

The $10 \mathrm{~min}$ were split into two $5 \mathrm{~min}$ recordings and hence 60 different data sets were used for the simulation runs of each mode. For model 2 and 3, basic frequencies for the sympathetic and the parasympathetic branch of the ANS needed to be determined. Therefore, a Lomb-Scargle periodogram of the patients RRintervals was calculated. The sympathetic frequency is chosen as the frequency with the highest power in the interval from $0.04-0.15 \mathrm{~Hz}$. The same applies for the parasympathetic activity and the frequency band from $0.15-0.4 \mathrm{~Hz}$.

For the solution of the various non-stiff differential equations, the ode 45 solver with variable step size was used. Every simulation was performed for $1000 \mathrm{sec}-$ onds, from which only the last 300 seconds were selected.

\section{Results}

Poincaré Plots The Poincaré plots of the first models 60 simulation runs clearly show a tendency of overestimation of the mean HR. This is apparent through the shift of the whole set of points along the line of identity, compared to the given patient data.

Another significant mismatch between given data and simulated data lies in the general shape of the point cloud. A typical distribution of points has a comet-like shape, whereas the simulated data presents itself in a more circular shape without any points in the centre of the point cloud. 


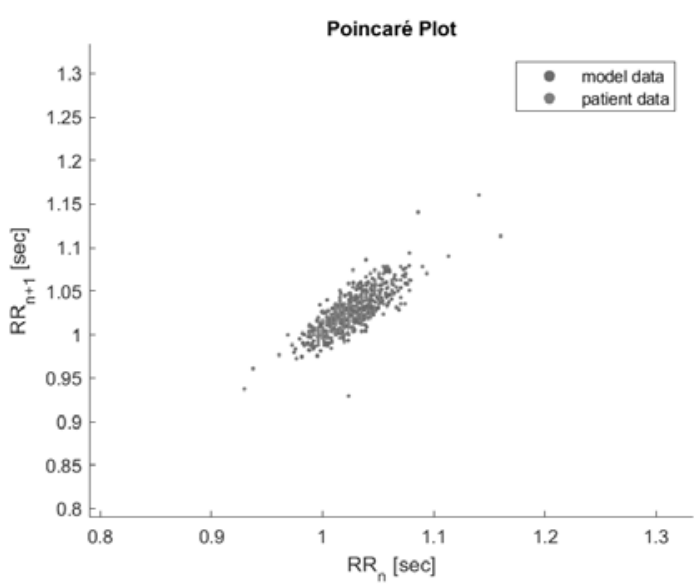

Figure 2: Model 2: Poincaré plot of the first 5 min recording of subject number 23 , with corresponding simulation output.

For the second model, considerably better Poincare plots could be achieved, especially in relation to the mean HR (Figure 2). For some cases however, the simulation output resulted in an atypical, elliptically appearing line. This phenomenon occurred mostly in connection with input data showing a rather large general dispersion of points.

The third model showed similar results concerning the mean HR, but a tendency of overestimation of the plots length and width was observed. For a variety of data sets, the plot showed a lack of points in the centre of the point cloud, although far less pronounced in comparison to the first model.

Statistical HRV Analysis Six statistical parameters were used for the quantification of the RR-data $[28,19]$. The mean RR- duration was analysed, followed by SD1, RMSSD, and SDSD as indicators for short term variability, SD2 as quantifier for long term variability and SDNN, which represents overall HRV.

As already indicated, model 1 tends to overestimate the RR-interval length drastically. For model 2 and 3, a very good replication of mean RR-duration could be reached. As shown in Figure 3, the results for the SDSD show a similar pattern to that of the mean RR-intervals. Again, the first model clearly overestimates the SDSD, opposite to the second and third model, which show a considerably better fit. When looking at the differences it should be noted, that the second model shows a slight underestimation, whereas the third model overestimates the SDSD a little.

SD1 shows the same behaviour as SDSD concerning the boxplot. Model 1 and 2 show a comparable amount of underestimation of SD2, which quantifies slow HRV changes, as opposed to a considerable overestimation by model 3. Again this behaviour is also observable in the Poincaré plots of model 3, as an overly long extension of the model data along the line of identity.

The RMSSD, which reflects high frequency components, basically shows the same behaviour as the previously described measures of short term variability.

SDNN, which is a measure of total spectral power, is most accurately represented by the output of the first model, slightly underestimated by the second model and clearly overestimated by the third.

Blood Pressure For Model 1 and 2, blood pressure showed a highly unusual behaviour for a remarkable number of cases. Systolic blood pressure mostly ranged above $140 \mathrm{mmHg}$, reaching maximum values of up to $190 \mathrm{mmHg}$.

This behaviour has a severe impact on the sympathetic activity, which is mainly dependent on the blood pressure function and its first derivative. Therefore, once systolic blood pressure levels exceed $160 \mathrm{mmHg}$, sympathetic activity shows phases of zero activity or even vanishes completely.

Another noteworthy result are the rapid changes in systolic, as well as diastolic blood pressure over the course of a few successive heartbeats. The systolic blood pressure varies by up to $20 \mathrm{mmHg}$ for both models, although less pronounced for the first than the second model. This is due to the impact of sympathetic nervous activity on the cardiac noradrenaline concentration.

Another cause of blood pressure fluctuations of about $10 \mathrm{mmHg}$ in both models is presented by respiratory activity. The changes in pressure are time-delayed in a way, that the peak in blood pressure appears shortly before a maximum level of inhalation is reached.

For the third model, during none of the simulation runs, a systolic blood pressure higher than $130 \mathrm{mmHg}$ was reached, and it generally showed only small fluctuations $(1-5 \mathrm{mmHg})$ over time. For the diastolic blood pressure, normal values were observed, with two exceptional cases of $40 \mathrm{mmHg}$ and $50 \mathrm{mmHg}$. In contrast to the systolic blood pressure, diastolic values showed quick changes of up to $10 \mathrm{mmHg}$ over the course of only a few heartbeats. 


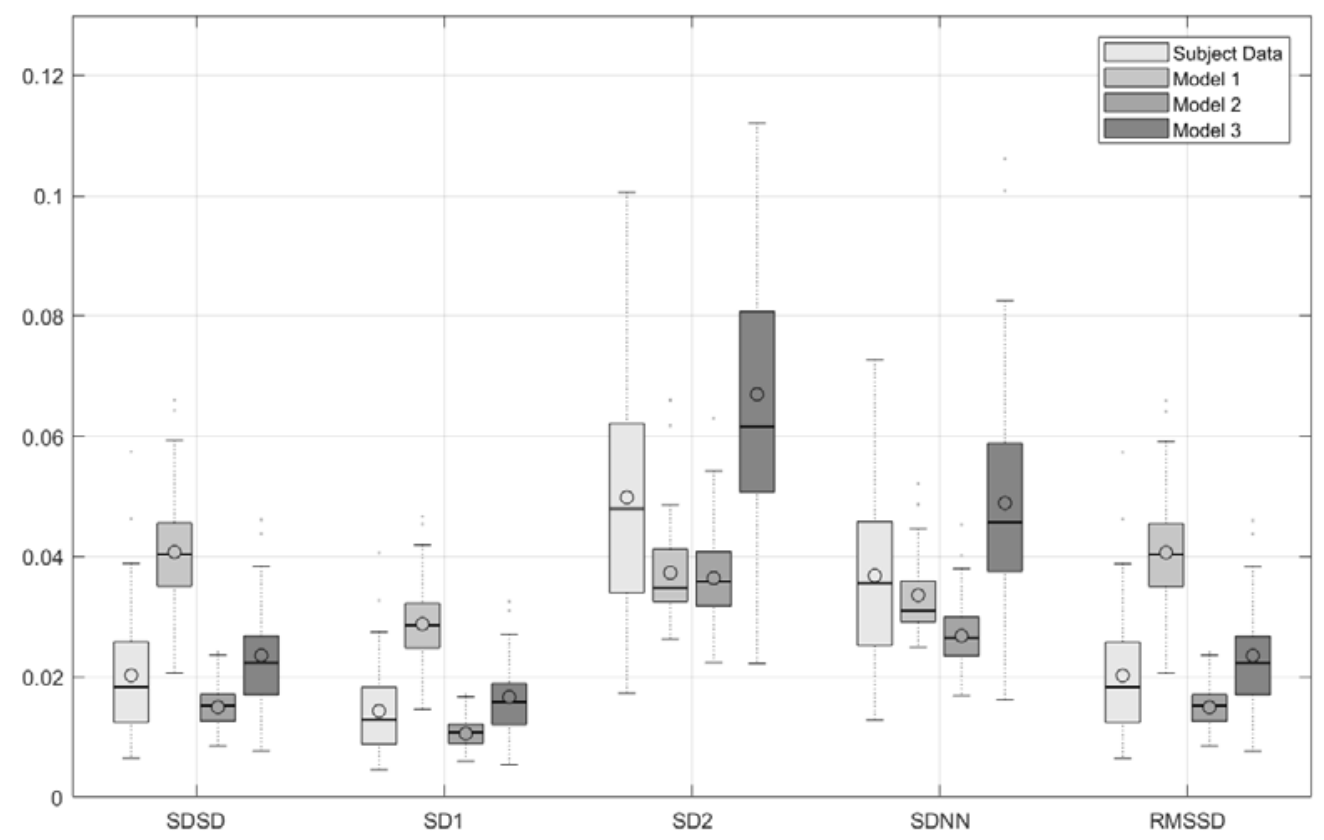

Figure 3: Boxplot of SDSD, SD1, SD2, SDNN, and RMSSD of the subject data and all three models.

Another notable observation about the behaviour of the blood pressure is its dependence on respiration. For the third model, respiration is included via variation of the IPFM threshold. It has an instantaneous influence on the occurrence time of the next heart beat, and consequently on blood pressure. For the first and second model, blood pressure variations can be observed, but due to the fact that respiration is included into the models ANS part, the time delay in sympathetic and parasympathetic activity results in a time delay between respiration and blood pressure variations.

\section{Discussion}

The Integral Pulse Frequency Modulation Model The first and most obvious result was the significant overestimation of the mean HR by the first model. This may be due to the fact that, although IPFM models are widely used in literature, they neglect respiration as an input, and their input functions are often designed to mimic physiological processes, but are not further mathematically analysed as stated by Meste et al. [29]. The averaged ANS IPFM input should equal 1 over the whole simulation run, in order to correctly recreate the mean HR. Mean HR should be additionally considered when modeling HRV, as it correlates with
HRV [30].

The third model showed a similar problem. For the second and third model, the introduction of a scaling function did indeed improve the results considerably, but this approach does not address the root of the problem.

Still, if the mean amplitude of the IPFM model input is, for example, too low, and therefore upscaled, the amplitude of the periodic input components is also increased, resulting in greater fluctuations. The change in amplitude of periodic signal parts has notable effects on the shape of the Poincaré plot and the measures of HRV. For the third model, the input of the IPFM was generally underestimated. By upscaling it, fluctuations of the incoming signal were reinforced, resulting in a greater overall variability. Moreover, the low frequency input signal components are dampened by this kind of signal modification, which makes the approach unsuitable for use in modeling and simulation of HRV over longer periods of time.

The Baroreceptors Seidel and Herzel state, that it is not reasonable to put much effort into baroreceptor modeling [2], but if all following model equations build on it, a more detailed approach is desirable at this point. Although baroreceptors can be seen as pro- 
portionally and differentially reacting controllers, other factors were ignored, such as adaption to changes in mean pressure, saturation, and neural conduction times. They could easily be included, as for example DeBoer et al. showed, using a scaled arctangent function to mimic saturation at high and low pressure levels [11].

For the third model, three different types of baroreceptors were presented, in order to account for the variation in thresholds for different baroreceptor types. They also include a maximum firing rate, which is a necessary assumption, since the firing rate is generally limited by the duration of the absolute refractory phase of baroreceptor ion channels. Their dependency on mean blood pressure over the last 1,5 , or 250 seconds gives them an adaptability to longer phases of hypertension, clearly missing in the first model[16]. Moreover, this modeling approach still presents an easily implementable, yet physiologically much more accurate basis for the input of the ANS, since at least two different types of baroreceptors are known [31].

ANS Activity The first model includes the basic characteristics of sympathetic and parasympathetic activity. They both show an oscillating behaviour and are modulated by baroreceptor activity, giving them a seemingly antagonistic behaviour. The basic frequency of both branches of the ANS was set equal to the respiratory frequency, which is contrary to the fact, that respiratory changes in HR were shown to be modulated mainly by parasympathetic activity [15]. The first model also modulates sympathetic activity proportional to the current baroreceptor activity, which is an assumption incompatible to the fact, that sympathetic activity is widely attributed to the low frequency components of HRV [28]. The erroneous regulation of sympathetic activity is also evident, if one considers that a maximum function had to be included into the model, in order to prevent negative sympathetic activity values. Another shortcoming lies in the complete absence of autonomous ANS activity independent from modulating factors.

Therefore, two oscillators for fundamental ANS activity, a low pass filter for sympathetic activity, and a term for respiration only in the parasympathetic nervous activity were included in the second model. The simulation results were superior to those of the first one by far, but still undesirable phases of zero sympathetic activity occurred, resulting in a lack of HRV. This leads to the conclusion, that baroreceptor activity and respi- ratory influences should not be included directly into sympathetic activity, if one locates sympathetic activity in the low frequency spectrum.

For the third model, the absence of independent ANS activity was analogously evened out by the inclusion of two oscillators. Since sympathetic activity was again directly dependent on the baroreceptor firing rate, another low pass filter was included. This causes a reaction to quick changes in blood pressure mainly mediated via the parasympathetic nervous system, which is consistent since this ANS part is attributed to the high frequency components of HRV.

Respiration Respiration, as a main influence on short term HRV, was included into the models in two different ways: The first and second model include respiration in the ANS, which is comprehensible, since changes in parasympathetic activity exist due to central respiratory modulation [15]. A disadvantage lies in the resulting temporal shift between inspiration and augmentation of the HR. The third model avoids this by varying the IPFM threshold based on the current level of in- and exhalation, leading to an instantaneous change of HR. It still is questionable, whether respiratory sinus arrhythmia should be modelled via the parasympathetic nervous system, or directly influence HR.

Blood Pressure Effective blood pressure regulation serves as an indicator of how well the model is constructed, since controlling mechanisms regulate the HR with the aim of keeping blood pressure levels within a normal range. In Studies, systolic blood pressure was shown to increase during phases of higher HR, whereas diastolic blood pressure was only slightly augmented in all age groups and for all heart rates [32]. The first and second model show significant changes in systolic, as well as diastolic blood pressure. Additionally, overestimated average blood pressure levels cause the previously mentioned phases of zero sympathetic activity. This is clearly not a desirable outcome and can be attributed to a combination of inadequacies. Quick changes in blood pressure during systole are normally evened out by the windkessel effect of the aorta. Although the first and second model do include a windkessel time constant, it only effects the diastolic pressure decrease. During systole, only the contractility varies based on sympathetic activity. This combination results in overly high blood pressure levels. Also, stroke volume and its dependence on the HR was not included, 
which further increases blood pressure levels. In comparison to the first two models, the third one showed a very stable mean systolic blood pressure for all patients and all mean heart rates, whereas the diastolic pressure showed fluctuations. The model expansion including HR dependent stroke volume and an arterial windkessel clearly had a stabilising effect on blood pressure. Especially the windkessel regulates blood pressure during systole, as well as diastole, and gives a more physiologic shape to the pressure curve. Although the third model behaves more realistical than the other two, correct selection of compliance and resistance is essential for an accurate calculation of blood pressure. Furthermore, fluctuations in rapid changes in diastolic blood pressure should be avoided generally. What is missing in the third model, is not only stroke volume, but also contractility depending on the filling of the heart. Varying peripheral resistance over time also presents a possibility for further model extension.

\section{Conclusion}

Although lots of existing models and submodels of the CVS include a variety of regulatory mechanisms of the HRV, they reveal shortcomings when closer examined. A lot of them do not address physiological processes in detail, or are largely based on purely statistical evaluations.

The first implemented model includes a variety of regulatory mechanisms, such as baroreceptors, sympathetic and parasympathetic activity, neurotransmitter concentrations, and myocardial contractility, but despite the many regulatory mechanisms it failed to mimic even the mean HR of the given subject data correctly.

The second model, which resulted from enhancements based on medical considerations of the first one, showed significant improvements in model performance when compared to subject data. However, it still generates physiologically untenable outputs for some cases. Phases of zero sympathetic activity caused by poor modeling of the baroreceptors, high fluctuations in systolic and diastolic blood pressure, and an incomplete arterial windkessel still provide starting points for further model enhancements.

The third model, being a combination of physiologically well-founded models, includes a more accurate description of the baroreceptors, and was also extended to further enhance its performance. Nevertheless, it overestimates short term as well as long term variabil- ity, which may be due to an inadequate choice of input for the IPFM model. In order to adequately recreate the mean HR given by the subject data, the IPFM input had to be evened out by a correction term analogously to the one used for the second model. In summary, models of cardiovascular regulation with an emphasis of correct represenation of HRV do exist, but only some of them are able to mimic reality to a satisfying degree. Modeling approaches, although physiologically valid, often put an emphasis on single aspects of the cardiovascular system, and simultaneously neglect others, which leads to a limited function of the whole model.

Once the existing regulatory mechanisms are accurately modelled, further enhancements may include age, gender, chemoreceptors, posture, hormonal regulation or even parts of the central nervous system. Nevertheless, further research needs to find a balance between a models richness of detail and an excessive amount of uncertain parameters.

\section{References}

[1] McCraty R, Shaffer F. Heart rate variability: new perspectives on physiological mechanisms, assessment of self-regulatory capacity, and health risk. Global Advances in Health and Medicine. 2015;4(1):46-61.

[2] Seidel H, Herzel H. Modelling heart rate variability due to respiration and baroreflex. In: Modelling the Dynamics of Biological Systems, pp. 205-229. 1995;.

[3] Acharya UR, Joseph KP, Kannathal N, Min LC, Suri JS. Heart rate variability. In: Advances in cardiac signal processing, pp. 121-165. 2007;

[4] McCorry LK. Physiology of the autonomic nervous system. American journal of pharmaceutical education. 2007;71(4):78.

[5] Pape HC, Kurtz A, Silbernagl S. Physiologie. 2018.

[6] Guyton A, Hall J. Textbook of medical physiology, 11th. 2006.

[7] Yasuma F, Hayano Ji. Respiratory sinus arrhythmia: why does the heartbeat synchronize with respiratory rhythm? Chest Journal. 2004;125(2):683-690.

[8] Teich MC, Lowen SB, Jost BM, Vibe-Rheymer K, Heneghan C. Heart rate variability: measures and models. arXiv preprint physics/0008016. 2000;.

[9] Barbieri R, Matten EC, Alabi AA, Brown EN. A point-process model of human heartbeat intervals: new definitions of heart rate and heart rate variability. American Journal of Physiology-Heart and Circulatory Physiology. 2005;288(1):H424-H435. 
[10] McLernon D, Dabanloo NJ, Ayatollahi A, Majd VJ, Zhang $\mathrm{H}$. A new nonlinear model for generating RR tachograms. In: Computers in Cardiology, 2004. IEEE. 2004; pp. 481-484.

[11] DeBoer RW, Karemaker JM, Strackee J. Hemodynamic fluctuations and baroreflex sensitivity in humans: a beat-to-beat model. American Journal of Physiology-Heart and Circulatory Physiology. 1987; 253(3):H680-H689.

[12] Mateo J, Laguna P. Improved heart rate variability signal analysis from the beat occurrence times according to the IPFM model. IEEE Transactions on Biomedical Engineering. 2000;47(8):985-996.

[13] Bailón R, Laouini G, Grao C, Orini M, Laguna P, Meste $\mathrm{O}$. The integral pulse frequency modulation model with time-varying threshold: application to heart rate variability analysis during exercise stress testing. IEEE transactions on biomedical engineering. 2011; 58(3):642-652.

[14] Brennan M, Palaniswami M, Kamen PW. A new cardiac nervous system model for heart rate variability analysis. In: Engineering in Medicine and Biology Society, 1998. Proceedings of the 20th Annual International Conference of the IEEE. IEEE. 1998; pp. 349-352.

[15] Eckberg DL. Point: counterpoint: respiratory sinus arrhythmia is due to a central mechanism vs. respiratory sinus arrhythmia is due to the baroreflex mechanism. Journal of applied physiology. 2009;106(5):1740-1742.

[16] Olufsen MS, Tran HT, Ottesen JT, Lipsitz LA, Novak V. Modeling baroreflex regulation of heart rate during orthostatic stress. American Journal of Physiology-Regulatory, Integrative and Comparative Physiology. 2006;291(5):R1355-R1368.

[17] Ursino M. Interaction between carotid baroregulation and the pulsating heart: a mathematical model. American Journal of Physiology-Heart and Circulatory Physiology. 1998;275(5):H1733-H1747.

[18] Kamen PW, Krum H, Tonkin AM. Poincare plot of heart rate variability allows quantitative display of parasympathetic nervous activity in humans. Clinical science. 1996;91(2):201-208.

[19] Brennan M, Palaniswami M, Kamen P. Do existing measures of Poincare plot geometry reflect nonlinear features of heart rate variability? IEEE transactions on biomedical engineering. 2001;48(11):1342-1347.

[20] Bazan O, Ortiz JP. Duration of Systole and Diastole for Hydrodynamic Testing of Prosthetic Heart Valves: Comparison Between ISO 5840 Standards and in vivo Studies. Brazilian journal of cardiovascular surgery. 2016;31(2):171-173.
[21] Aysin B, Aysin E. Effect of respiration in heart rate variability (HRV) analysis. In: Engineering in medicine and biology society, 2006. EMBS'06. 28th annual international conference of the IEEE. IEEE. 2006; pp. 1776-1779.

[22] Westerhof N, Lankhaar JW, Westerhof BE. The arterial windkessel. Medical \& biological engineering \& computing. 2009;47(2):131-141.

[23] Parragh S. Modelle zur Bestimmung des aortalen Blutflusses basierend auf Optimalitätsbedingungen. Master's thesis, TU Wien, Institut für Analysis und Scientific Computing. 2013.

[24] Boudoulas H, Geleris P, Lewis RP, Rittgers SE. Linear relationship between electrical systole, mechanical systole, and heart rate. Chest. 1981;80(5):613-617.

[25] Van de Vooren H, Gademan MG, Swenne CA, TenVoorde BJ, Schalij MJ, Van der Wall EE. Baroreflex sensitivity, blood pressure buffering, and resonance: what are the links? Computer simulation of healthy subjects and heart failure patients. Journal of Applied Physiology. 2007;102(4):1348-1356.

[26] Barbi M, Chillemi S, Garbo AD, Balocchi R, Menicucci D. A minimal model for the respiratory sinus arrhythmia. Biological cybernetics. 2006; 94(3):225-232.

[27] Bachler M, Sehnert W, Mikisek I, Mengden T, Wassertheurer S. Abstract 15861: Device-Guided Slow Breathing With Real-Time Biofeedback for Personalized Cardiovascular Therapy Support Increases Pulse Transit Time. Circulation. 2018; Vol 138.

[28] Malik M. Heart rate variability. Annals of Noninvasive Electrocardiology. 1996;1(2):151-181.

[29] Meste O, Khaddoumi B, Blain G, Bermon S. Time-varying analysis methods and models for the respiratory and cardiac system coupling in graded exercise. IEEE transactions on biomedical engineering. 2005;52(11):1921-1930.

[30] Antelmi I, De Paula RS, Shinzato AR, Peres CA, Mansur AJ, Grupi CJ. Influence of age, gender, body mass index, and functional capacity on heart rate variability in a cohort of subjects without heart disease. The American journal of cardiology. 2004; 93(3):381-385.

[31] Lohmeier TE, Iliescu R. The baroreflex as a long-term controller of arterial pressure. Physiology. 2015; 30(2):148-158.

[32] Miyai N, Arita M, Miyashita K, Morioka I, Shiraishi T, Nishio I. Blood pressure response to heart rate during exercise test and risk of future hypertension. Hypertension. 2002;39(3):761-766. 